\title{
Effects of environmental noise on the accuracy of millimeter sized grippers in cantilever configuration and active stabilisation
}

\author{
Mokrane Boudaoud, Yassine Haddab, IEEE Member, Yann Le Gorrec, and Philippe Lutz, IEEE Member
}

\begin{abstract}
This paper presents a study about the effects of environmental noise on millimeter sized grippers in cantilever configuration. The study is motivated and conducted aiming at assessing the level of accuracy loss when performing micromanipulation/microassembly tasks in noisy environments as well in typical microrobotics laboratories as in industrial locations or operating rooms. Ground motion and acoustic noises within a typical microrobotic laboratory are characterized in the frequency domain and their effects on cantilevers of different lengths are inspected. The relevance of a typical vibration isolation table is evaluated and the effects of low and high acoustic noises are assessed. A modeling of a cantilever with base excitation is thereafter conducted in the state space using finite difference formulation and a stabilization of a disturbed cantilever is obtained at the nanometer level in noisy environments allowing perspectives to high precision micromanipulation tasks in hostile locations.
\end{abstract}

\section{INTRODUCTION}

Accurate and high precision micromanipulation tasks are required in a wide range of microrobotics applications such as microassembly [1], force sensing [2] and surgical operations [3]. In that sense, numerous microgrippers have been previously designed based on actuators endowed with high position resolutions and good deformation/force ratios allowing precise motions of microgrippers arms, such as the one used in our previous work [4]. However, despite high performances of actuators, the vibratory behaviour specific to the arms is considered as the main source of accuracy loss [5] [6] [7] prejudicing wanted performances in a given micromanipulation task. Unwanted vibrations are mostly due to the environmental noise such as ground motion and acoustic noises which can be produced by human activity, operating machines, fans, etc within a given location. Ground motion is often characterized by a complex spectrum consisting of fast and slow motions [8] [9] [10]. While slow motions relates on the own activity of the earth which is concentrated below few hertz, it is the cultural noise with frequencies higher than a few hertz that has the potential of affecting flexible structures and especially near their resonant frequencies by acting on clamped parts. Cultural noise relating mainly on operating machines has generally a significant energy from a few hertz up to a few hundred of hertz [8] and its frequential behavior can vary from one location to another. To deal with performance limitations caused by ground motion, microgrippers are often mounted on vibration isolations

M. Boudaoud, Y. Haddab, Y. Le Gorrec and P. Lutz are with the Automatic Control and Micro Mechatronic Systems Department, FEMTO-ST Institute, UFC-ENSMM-UTBM-CNRS, University of FrancheComté, Besançon, France. (mokrane.boudaoud, yassine.haddab, legorrec, philippe.lutz)@femto-st.fr tables and mechanical designs are sometimes studied to reduce the low frequency vibrations [11]. However, acoustic noises are rarely taken into account in micromanipulation tasks although this noise can have an important effect on mechanical structures [12] and can be over than $70 \mathrm{~dB}$ in typical operating rooms [13], while numerous microgrippers are devoted to surgical applications [14]. In this case, vibration isolation tables can be insufficient and acoustic isolation may be necessary for high precision operations. The study presented in this paper is conducted with the aim of assessing the level of accuracy loss within microgrippers when performing micromanipulation/ microassembly tasks in noisy environments as well in typical microrobotics laboratories as in industrial locations or operating rooms. As starting point, the focus is given to mechanical designs in cantilever configuration with millimeter sized dimensions wich is often encountered on the arms of microgrippers such as in [15], the developed microgripper is based on three arms in cantilever configuration $(40 \mathrm{~mm} \times 10 \mathrm{~mm} \times$ $0.2 \mathrm{~mm}$ ), also in [16] a piezoelectric cantilever is used for microassembly tasks with $15 \mathrm{~mm}$ length, $2 \mathrm{~mm}$ width and 0.3 $\mathrm{mm}$ thickness. As such, for the given study, cantilevers of

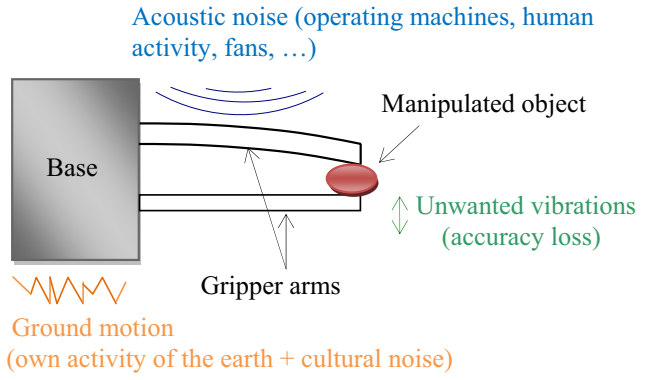

Fig. 1. Simplified scheme of a microgripper made up of two arms in cantilever configuration subject to environmental noise.

different lengths clamped on the same stiff support have been designed for experiments such as their first flexural resonance is located in low frequencies (few hundred of hertz) where ground motion and acoustic noises should be both relevant as stated in the literature. A frequential characterisation of these noises is then conduced in our laboratory to check precisely about the frequency range where they are significant and their effects on the designed cantilevers are inspected. For this purpose, effects of ground motion are studied in low acoustic noise conditions and the relevance of a vibration isolation table for vibration rejection is inspected. After that, effects of low and high acoustic noises are assessed when the experimental station is located on the vibration 
isolation table, in which a loudspeaker is used to generate $70 \mathrm{~dB}$ acoustic perturbations as in operating rooms for high acoustic noise case. The interest of this approach is to check for each condition, in which frequential domain a stabilisation of the cantilevers is needed aiming at improving position accuracy. A modeling of a cantilever with base excitation is thereafter conducted using the finite difference

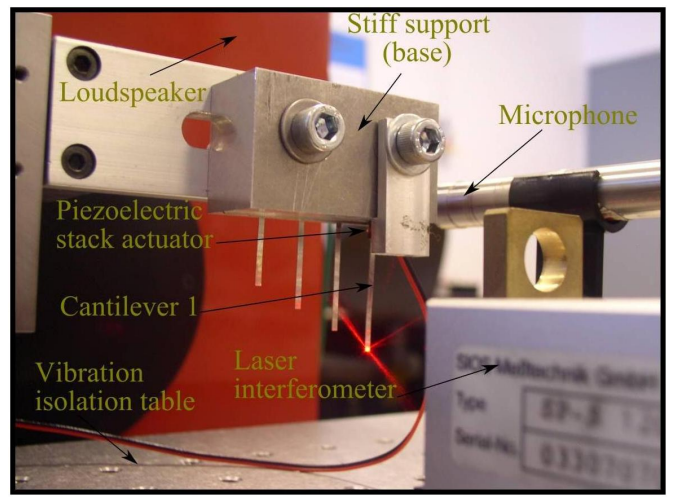

Fig. 2. Experimental station for noise analysis in a microrobotic laboratory.

approximation with an optimisation of dynamical parameters, leading to the development of a reliable state space model for an active vibration control. Finally, using a piezoelectric stack actuator and a high resolution laser interferometer, a stabilization of the most disturbed cantilever is obtained at the nanometer level while tracking sinusoidal reference in a noisy environment, allowing perspectives to high precision micromanipulation applications in hostile locations.

\section{DESCRIPTION OF THE EXPERIMENTAL STATION}

Starting from a bulk of aluminum, four cantilevers of different lengths (TABLE I) have been machined using a wire processing such as all cantilevers are clamped on the same stiff support (no machined part) (Fig. 2). Vibration measurements are performed using a high resolution $(0.01 \mathrm{~nm})$ laser interferometer sensor (SP-120 SIOS Mebtechnik GmbH) covering a frequency range from few hertz up to $1 \mathrm{MHz}$, while a microphone $(1 / 2$ " $50 \mathrm{mV} / \mathrm{Pa}, 01 \mathrm{~dB}$-metravib) connected on an amplifier (PRE21S, 01dB-Metravib) allowed acoustic noise measurements $(3.15 \mathrm{~Hz}$ up to $20 \mathrm{KHz})$. High acoustic noises are generated using a loudspeaker (LD 130 CR08 ATOHM) and a piezoelectric stack actuator (P-249.20, Physik Instrument $\mathrm{GmbH}$ ) with $20 \mathrm{KHz}$ resonant frequency is used

TABLE I

DIMENSIONS OF THE CANTILEVER BEAMS

\begin{tabular}{l||llll}
\hline & cantilever 1 & cantilever 2 & cantilever 3 & cantilever 4 \\
\hline Length & $30 \mathrm{~mm}$ & $25 \mathrm{~mm}$ & $20 \mathrm{~mm}$ & $15 \mathrm{~mm}$ \\
\hline Width & $1.5 \mathrm{~mm}$ & $1.5 \mathrm{~mm}$ & $1.5 \mathrm{~mm}$ & $1.5 \mathrm{~mm}$ \\
\hline Thickness & $0.22 \mathrm{~mm}$ & $0.22 \mathrm{~mm}$ & $0.22 \mathrm{~mm}$ & $0.22 \mathrm{~mm}$ \\
\hline
\end{tabular}

for the mechanical characterization of the cantilevers through base excitations. For small displacements (less than 50nm), experimental measurements showed that the stack actuator obeys to a linear supply voltage/displacement characteristic with a sensitivity equal to $K_{\text {act }}=1.6 \mathrm{~nm} / \mathrm{Volts}$.

\section{ENVIRONMENTAL NOISE ANALYSIS WITHIN A TYPICAL MICROROBOTIC LABORATORY}

\section{A. Ground motion and acoustic noise measurements}

Ground motion and acoustic noise have been measured in the microrobotic laboratory (Automatic Control and MicroMechatronic Systems Department, Femto-ST institute) during the day when all usual sources of noise have been present (running computers + fan + different amplifiers + experimental station for noise analysis) including low human activity relating only to people working on their stations and speaking together such as no ground motion measurements have been performed when people were walking on the floor. Considering that an important part of the motion of the ground is transmitted to the base of cantilevers, the

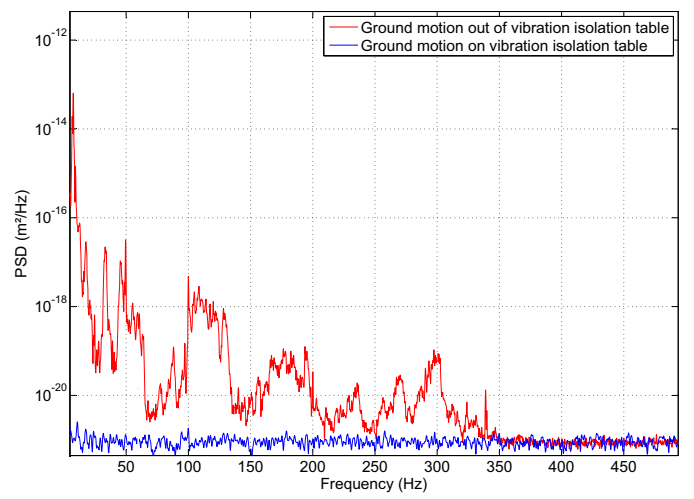

Fig. 3. PSD of ground motion within the microrobotic laboratory.

term "ground motion" has been assigned through misuse of language to the vibratory behaviour of the base. Then, the laser interferometer sensor has been used in order to perform horizontal vibration measurements on the base with $25 \mathrm{KHz}$ sampling frequency. Measurements have been performed during one hour, which is sufficient for the characterisation of fast motion [10]. Moreover, by way of comparison, measurements have been performed on a vibration isolation

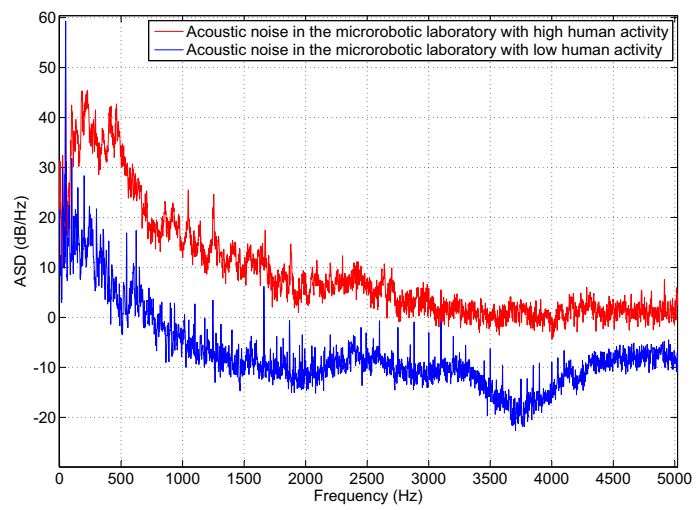

Fig. 4. ASD of acoustic noises within the microrobotic laboratory.

table (kinetic systems, vibraplane, model no 9101-21-46) and out of this last using a simple table located on the floor. On the other hand, acoustic noises have been measured during the day when three persons were present within 
the laboratory (case of low human activity), and secondly twelve persons talking together (not walking) have been added in order to check about the increase of acoustic noise level in high human activity conditions and also to assess whether the level of acoustic noise produced by humans can have a significant effect on the cantilevers. Each acoustic measurement has been performed during 60 seconds with 5208,33 Hz sampling frequency. Power and amplitude spectral densities (PSDs and ASDS) of noises have been averaged 128 times using the Welch method which is often recommended for the case of noisy signals [17]. As shown in (Fig. 3), the effectiveness of the vibration isolation table is clearly observed, such as on this table only the PSD of the internal laser interferometer noise is detected due to its resolution. Ground motion is in this case less than $0.01 \mathrm{~nm}$. However, out of the vibration isolation table, the PSD of ground motion generally decreases with increasing the frequency and vanishes beyond $350 \mathrm{~Hz}$ (below the resolution of the laser interferometer). The measured ground motion in this case is due to the cultural noise. For this reason and in order to keep the same frequential behavior, effects of ground motion are thereafter analysed by keeping the same sources of perturbations. On the other hand, through the frequential behavior of acoustic noises (Fig. 4), it is possible to observe that the threshold of audibility $(0 \mathrm{~dB})$ is reached at $500 \mathrm{~Hz}$ in low human activity, while it is about $3 \mathrm{KHz}$ in high human activity conditions. human speech can then cause an important increase of acoustic noise which is nevertheless the most significant below $1 \mathrm{KHz}$ in high human activity.

B. Effect of acoustic noise and ground motion on the cantilever beams within the microrobotic laboratory

Vibration measurements are performed at the free end of each cantilever to check about the effect of the environmental noise in the previous studied conditions. In this

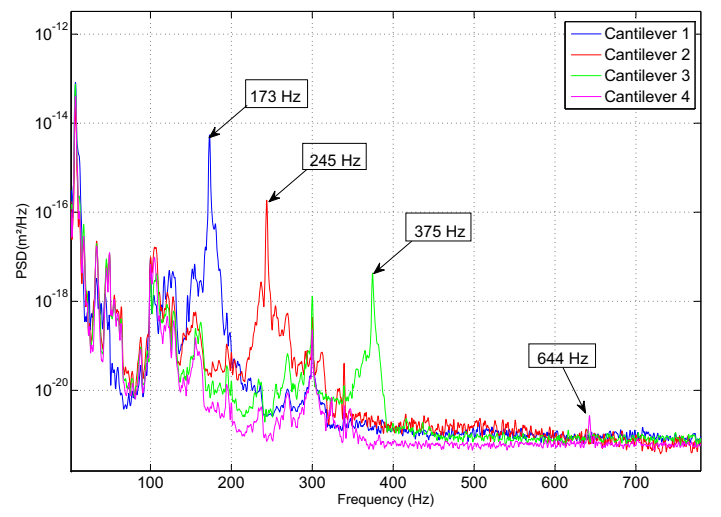

Fig. 5. PSD of cantilevers vibrations out of the vibration isolation table within the microrobotic laboratory.

case, a comparison between the vibratory behaviour of the cantilevers on and out of the vibration isolation table has been conducted with low human activity (Fig. 5), (Fig. 6). After that, measurements are only performed on the vibration isolation table (reducing the motion of the ground) in low and high human activity conditions for the analysis of the effect of acoustic noises. Moreover, in order to assess about the effects of acoustic noises which can be found in typical operating rooms, vibrations of the cantilevers have been also measured on the vibration isolation table while the loudspeaker was generating $70 \mathrm{~dB}$ sinusoidal acoustic noise exciting the first resonance of each cantilever.

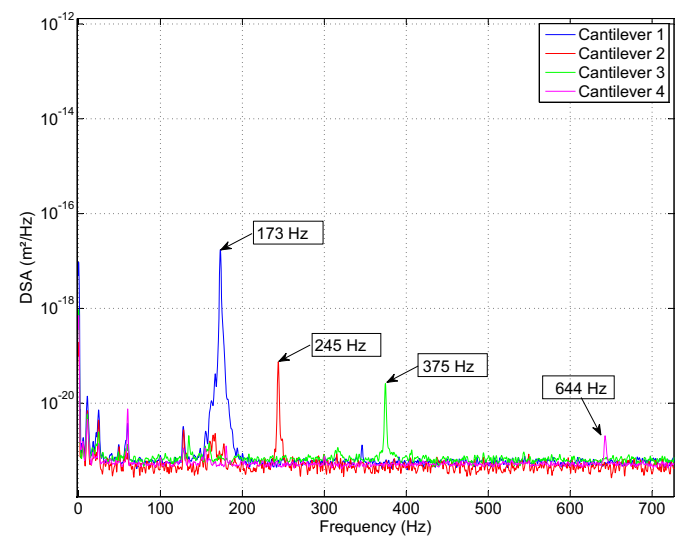

Fig. 6. PSD of cantilevers vibrations on the vibration isolation table within the microrobotic laboratory.

In all computed PSDs, only the first flexural resonance of each cantilever has been clearly observed, other resonances which are located above $1 \mathrm{KHz}$ were drowned in the interferometer noise. For this reason, and in order to get displacement values around the significant resonances, integrated RMS displacement have been extracted from each $\mathrm{PSD}$ in $\Delta f=f_{2}-f_{1}=50 \mathrm{~Hz}$ bandwidth around first resonance where the effect of the environmental noise is the most significant. This was conducted using the integrated RMS method such as [18] :

$$
R M S_{\text {int }}\left(f_{1}, f_{2}\right)=\sqrt{\sum_{f_{1}}^{f_{2}} P S D(f) \cdot \Delta f}
$$

In this study, only RMS displacements are presented (TABLE II). Note that the maximum displacement is higher than the RMS one. In this case, nanometer RMS displacements have then been measured at the free end of each cantilever due to the environmental perturbations which may represent a limitation if nanometer accuracy is required in a given micromanipulation task. Moreover, as it could be expected, the longer are the cantilevers, the more is the effect of environmental noise, while long cantilevers are more suitable for large displacements. The vibration isolation table is then important for high precision operations until the flexural resonance of the cantilevers is below frequencies neighbouring $350 \mathrm{~Hz}$ (see the case of cantilever 4). However due to other origin of noises such as acoustic ones, the given table may be not enough. Indeed, with the mere increase of the number of people talking in the laboratory, the RMS displacement of all cantilevers increased reaching ones measured out of this table in low human activity conditions. Also, vibrations reaching the micrometer level are observed for cantilevers 1 and 2 with the action of the loadspeaker $(70 \mathrm{~dB})$. In addition 
TABLE II

EFFECT OF THE ENVIRONMENTAL NOISE ON THE ALUMINUM CANTILEVERS : RMS DISPLACEMENT AT CANTILEVERS FREE END

\begin{tabular}{l|cccc}
\hline & cantilever 1 & cantilever 2 & cantilever 3 & cantilever 4 \\
\hline On the vibration isolation table with low human activity & $7.2 \mathrm{~nm}$ & $0.9 \mathrm{~nm}$ & $0.45 \mathrm{~nm}$ & $0.1 \mathrm{~nm}$ \\
\hline On the vibration isolation table with high human activity & $123.8 \mathrm{~nm}$ & $20 \mathrm{~nm}$ & $6 \mathrm{~nm}$ & $0.5 \mathrm{~nm}$ \\
\hline On the vibration isolation table with 70 dB acoustic disturbance (loadspeaker) & $1.8 \mu \mathrm{m}$ & $0.82 \mu \mathrm{m}$ & $200 \mathrm{~nm}$ & $85 \mathrm{~nm}$ \\
\hline Out of the vibration isolation table with low human activity & $112.8 \mathrm{~nm}$ & $19.1 \mathrm{~nm}$ & $5 \mathrm{~nm}$ & $0.4 \mathrm{~nm}$ \\
\hline
\end{tabular}

to the vibration isolation table, a stabilisation of cantilevers position may be necessarily to increase position accuracy and especially in the case of harsh environments. In that sense, the next section deals with a modelling of a cantilever with base excitation allowing the development of a reliable state space model for an active stabilisation of tip displacements. In this paper, modelling and control are treated only in the case of cantilever 1 . Nevertheless, the same work can be done on other cantilevers.

\section{DyNAMiC MODELling OF A CANTILEVER WITH BASE EXCITATION}

\section{A. Finite difference formulation of the Euler-Bernoulli can- tilever and state space representation}

Consider the partial differential equation of EulerBernoulli, describing the vibratory behaviour $v(x, t)$ of a beam subject to an applied force $F(x, t)$ per unit length :

$$
\text { E.I. } \frac{\partial^{4} v(x, t)}{\partial x^{4}}+\delta . \frac{\partial v(x, t)}{\partial t}+\rho . S . \frac{\partial^{2} v(x, t)}{\partial t^{2}}=F(x, t)
$$

Where $E$ is the Young modulus, $I$ is the area moment of inertia about $z$ axis, $\delta$ is the damping factor, $\rho$ is the mass density and $S$ defines the area of the transverse section.

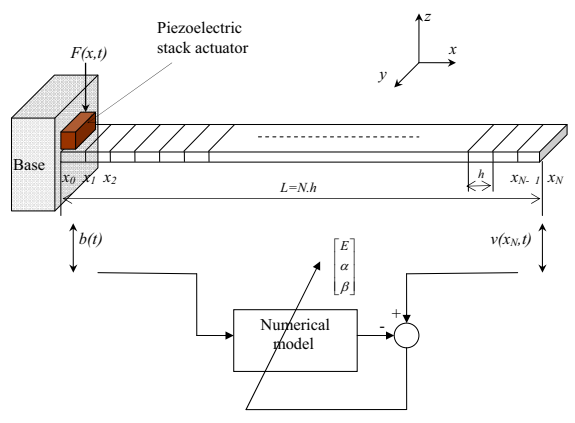

Fig. 7. Spatial discretization of a cantilever beam and optimal estimation of mechanical parameters.

In this study, partial derivatives in spatial dimension are approximated with the central finite difference scheme, using $N$ grid points (nodes) separated by a length of $h$ on the beam (Fig. 7) where $v\left(x_{n}\right)$ designates the deflexion of the beam at a location $n \times h$. For the cantilever case with a base excitation expressed by the variable $b(t)$, the boundary conditions at both ends of the flexible structure are defined as [19]:

$$
\left\{\begin{array}{l}
v\left(x_{0}, t\right)=b(t)=\frac{h^{4}}{E \cdot I} \cdot F(t) \quad\left\{\begin{array}{l}
v^{\prime \prime}\left(x_{N}, t\right)=0 \\
v^{\prime \prime \prime}\left(x_{N}, t\right)=0
\end{array}\right. \\
v^{\prime}\left(x_{0}, t\right)=0
\end{array}\right.
$$

The partial differential equation (2) can be converted into state space representation, for which the input of the model is granted to the base motion while the output relates to the displacement of the cantilever free end, such as :

$$
\begin{aligned}
& \left\{\begin{array}{l}
\dot{X}(t)=A \cdot X(t)+B \cdot b(t) \\
v\left(x_{N}, t\right)=C \cdot X(t)
\end{array}\right. \\
& X=\left[\begin{array}{ll}
V & \dot{V}
\end{array}\right]^{T}, \\
& A=\left(\begin{array}{cc}
0_{N} \times N & I_{N} \\
-\left(\rho \cdot S \cdot I_{N}\right)^{-1} \cdot \frac{E \cdot I}{h^{4}} \cdot(\text { deriv } 4) & -\left(\rho . S \cdot I_{N}\right)^{-1} \cdot \delta \cdot I_{N}
\end{array}\right) \text {, } \\
& B=\left(\begin{array}{c}
0_{N \times 1} \\
\left(\rho \cdot S \cdot I_{N}\right)^{-1} \cdot M_{f}
\end{array}\right), C=\left(\begin{array}{cccc}
0_{1 \times(N-1)} & 1 & 0 & 1 \times N
\end{array}\right) \\
& A \in \Re^{2 N \times 2 N}, B \in \Re^{2 N \times 1} \text { and } C \in \Re^{1 \times 2 N} \text {. }
\end{aligned}
$$

$I_{N} \in \mathbb{R}^{N \times N}$ is the identity matrix

The load matrix $M_{f}$ and the fourth derivative matrix [derive 4] are derived from finite difference formulation with a respect of the boundary conditions, such as :

$$
\begin{gathered}
M_{f}=\frac{E \cdot I}{h^{4}} \cdot(-6,4,-1,0, \ldots, 0)^{T} \\
{[\text { derive }]=\left(\begin{array}{cccccccc}
0 & -8 & 1 & 0 & & \ldots & & 0 \\
0 & 7 & -4 & 1 & 0 & \ldots & \ldots & 0 \\
1 & -4 & 6 & -4 & 1 & 0 & \ldots & 0 \\
0 & \ddots & \ddots & \ddots & \ddots & \ddots & \ddots & \vdots \\
\vdots & 0 & 1 & -4 & 6 & -4 & 1 & 0 \\
0 & \cdots & 0 & 1 & -4 & 6 & -4 & 1 \\
0 & & \cdots & 0 & 1 & -4 & 5 & -2 \\
0 & & \cdots & & 0 & 2 & -4 & 2
\end{array}\right)}
\end{gathered}
$$

The developed model is only an approximation of the dynamics of the actual system, theoretical approach must be then compared with experiments and especially for the damping around the first flexural resonance. For further details about finite difference formulations, the reader can refer to [20].

\section{B. Damping formulation and numeric model validation}

According to $N=512$ grid points in the discretized scheme, the damping matrix has been computed using Lord Rayleigh's hypothesis [21], for which :

$$
\delta . I_{N}=\alpha \cdot\left(\frac{E . I}{h^{4}} \cdot[\text { derive } 4]\right)+\beta \cdot\left(\rho . S . I_{N}\right)
$$

Weighting parameters $\alpha$ and $\beta$ can be extracted by defining damping ratios of two resonant modes [21]. However, in our study we have chosen to perform an optimization of weighting parameters with a least squares identification method (Levenberg- Marquardt algorithm) using experimental data. Moreover, in order to get through the model a first resonant frequency as close as the experimental one, the Young modulus has been also used as a parameter to be optimized 
using its theoretical value $E=67 G P a$ as initialisation. The mass density in this case has been fixed at $\rho=2900 \mathrm{Kg} / \mathrm{m}^{2}$. Using the piezoelectric stack actuator fixed near the clamped part of the cantilever 1 , a 1 Volts step excitation has been applied and the corresponding free end response has been used for parameters optimization (Fig. 7). Thus, optimal parameters leading to a minimum value of error between the output of the model and the experimental data have then been defined as : $E=67.15 G P a, \alpha=2.31 .10^{-7}$ and $\beta=1.3572$.

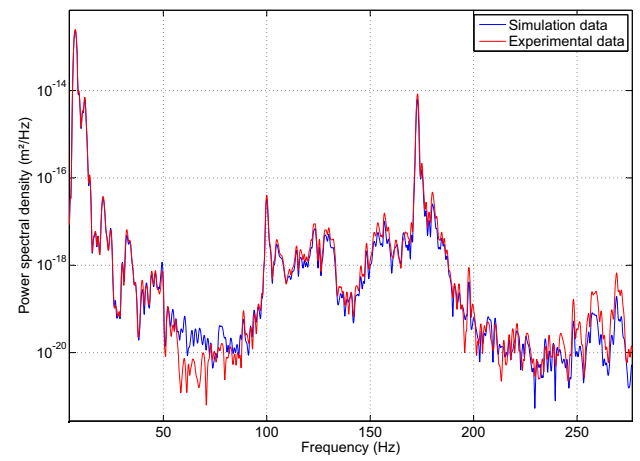

Fig. 8. PSD of cantilever 1 vibrations subject to ground motion

For model validation, the measured ground motion remaining out of the vibration isolation table (Fig. 3) with low human activity has been used as an input signal to the numerical model and the output which relate to the vibrations of Nth node of the discretized cantilever (free end) has been extracted to compute PSD. Results are presented in (Fig. 8) through which the effectiveness of the model can be observed. An active controller for vibration rejection can be designed based on the developed model, aiming at improving the accuracy of the cantilever in the nanometer level.

\section{ACtive STABILIZATION OF THE CANTILEVER 1 SUBJECT TO NOISE DISTURBANCES}

In addition to the vibration isolation table, a stabilization of the cantilever 1 free end position at the nanometer level is discussed through active noise control. An optimal linear quadratic LQ discret controller is suggested.

\section{A. Discrete state space model for active control}

Starting from the previous designed model containing 1024 states, an order reduction has been performed for the control part. Based on implicit balancing techniques to compute the reduced- order approximation [22], a forth order model has been retained, and for $10 \mathrm{KHz}$ sampling frequency, the discrete form of the state space model is given as :

$$
\left\{\begin{array}{l}
X_{d}(k+1)=A_{d} \cdot X_{d}(k)+B_{d} \cdot b_{d}(k) \\
v_{d}\left(x_{N}, k\right)=C_{d} \cdot X_{d}(k)
\end{array}\right.
$$

$$
\begin{aligned}
A_{d} & =\left(\begin{array}{cccc}
0.9940 & 0.1088 & 0 & 0 \\
-0.1088 & 0.9940 & 0 & 0 \\
0 & 0 & 0.7753 & 0.6306 \\
0 & 0 & -0.6306 & 0.7753
\end{array}\right), \\
B_{d} & =\left(\begin{array}{cccc}
0.0114 & 0.1847 & -0.4693 & 0.1670
\end{array}\right)^{T}, \\
C_{d} & =\left(\begin{array}{llll}
0.6705 & -0.0048 & -0.0010 & -0.2438
\end{array}\right) .
\end{aligned}
$$

Moreover, since the actuator is characterized by a high resonant frequency comparing to the one specific to the cantilever, its dynamic has been neglected in the feedback control design, such as only the sensitivity $K_{a c t}$ (static case) is considered in the model.

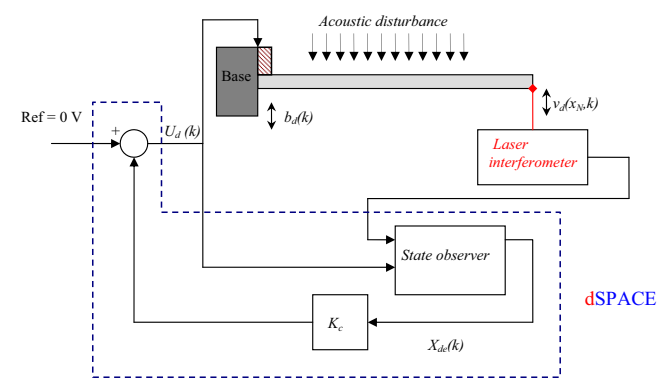

Fig. 9. Block diagram of the closed loop control system.

\section{B. Optimal controller design and real time implementation}

The piezoelectric stack actuator has been used to generate base excitations following the control low and aiming at counteracting cantilever free end vibrations which are measured in real time by the laser interferometer. The optimal controller is calculated on the basis of the reduced state space model in such a way that the feedback law $U_{d}(k)=$ $b_{d}(k) \cdot K_{a c t}^{-1}=-K_{c} \cdot X_{d}(k)$ minimizes the performance criteria $J(k)$ in which $Q$ and $R$ are positive definite matrices.

$$
J(k)=\sum_{i=k}^{\infty} X_{d}^{T}(i) \cdot Q \cdot X_{d}(i)+U_{d}^{T}(i) \cdot R \cdot U_{d}(i) .
$$

For a discrete computation of the optimal controller :

$$
K_{c}=\left(R+\left(B_{d} \cdot K_{a c t}\right)^{T} \cdot P_{c} \cdot B_{d} \cdot K_{a c t}\right)^{-1} \cdot\left(B_{d} \cdot K_{a c t}\right)^{T} \cdot P_{c} \cdot A_{d}
$$

$P_{c}$ is the solution of Riccati equation and can be calculated from matlab software using dare function for which weighting parameters $R$ and $Q$ must be beforehand selected. Moreover, a conventional Luenberger observer has been added allowing the estimation of the state variable $X_{d e}(k)$ such as :

$$
X_{d e}(k+1)=F_{d} \cdot X_{d e}(k)+B_{d} \cdot K_{a c t} \cdot U_{d}(k)+K_{o} \cdot v_{d}\left(x_{N}, k\right)
$$

Where $F_{d}=\left(A_{d}-K_{o} . C_{d}\right)$, and $K_{o}$ is the gain of the observer used such as the discrete error $e_{d}(k)=X_{d}(k)-X_{d e}(k)$ converge asymptotically to zero with a dynamic higher than the one of the closed loop system. As such $K_{o}$ has been chosen such as the discrete error converge to zero with a response time ten times smaller than the one of the closed loop system, which resulted :

$$
K_{o}=\left[\begin{array}{llll}
8.3573 & 21.6619 & 7.3919 & 9.2190
\end{array}\right]^{T}
$$

For vibration rejection, weighting parameters of the optimal criterion have been selected experimentally leading to :

$$
R=10^{-9} \text { and } Q=\operatorname{diag}\left(10^{9}, 1,10^{9}, 1\right)
$$

Thus : $K_{c}=10^{8} \cdot\left[\begin{array}{llll}3.1737 & 5.2338 & -6.0746 & -0.6704\end{array}\right]$ 

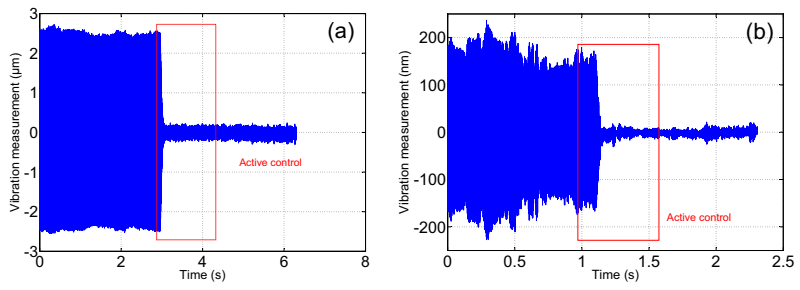

Fig. 10. Active control of cantilever 1 subject to $70 \mathrm{~dB}$ acoustic noise disturbance (a) and in high human activity conditions (b).

The control algorithm has been developed using the MATLAB/Simulink software and implemented in real time with a dSPACE (RTI1104) control board running at $10 \mathrm{KHz}$ sampling frequency. Experimental results are presented in (Fig. 10 (a)) for vibrations rejection when the cantilever 1 is subject to 70 $\mathrm{dB}$ acoustic noise, and in (Fig. 10 (b)) in high human activity condition. Moreover in (Fig. 11), it possible to observe the improvement of the cantilever accuracy while tracking 15 $\mathrm{nm}$ sinusoidal reference with $1 \mathrm{~Hz}$ frequency in high human acoustic noise.

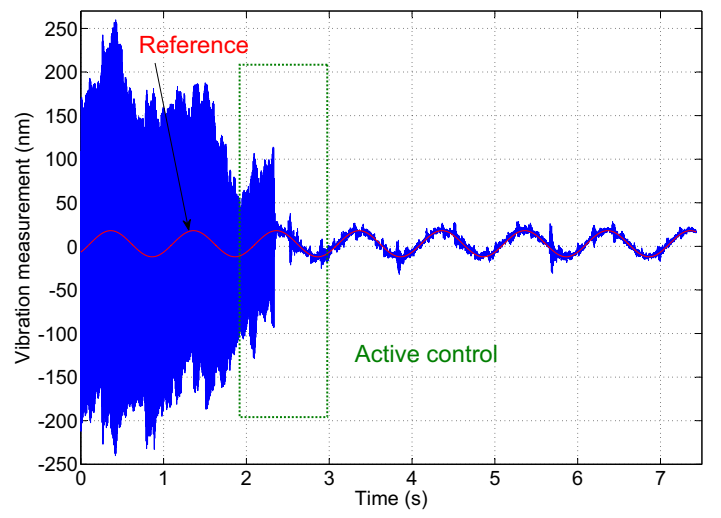

Fig. 11. Active control of cantilever 1 while tracking $15 \mathrm{~nm}$ sinusoidal reference at $1 \mathrm{~Hz}$ in high human activity conditions

\section{CONCLUSION}

In this paper, an inspection about the effects of environmental noise on millimeter sized cantilevers which may represent end effectors of micromanipulators have been performed with the aim of assessing the level of accuracy loss when working in noisy environments. Ground motion and acoustic noises within a typical microrobotics laboratory have been studied and characterized in the frequency domain. The vibration isolation table proved that it was not sufficient for high position accuracy when using microgrippers with low resonant frequencies (less than $1 \mathrm{KHz}$ ) and especially in the case of acoustic perturbations even produced by humans. Moreover, vibrations reaching the micrometer level have been observed in the case of operating rooms conditions. In this case, an optimal active vibration controller has been proposed to ensure a stabilisation of the most disturbed cantilever at the nanometer level in presence of acoustic perturbations. The effectiveness of the controller has been verified experimentally in the case of high human activity condition and while disturbing the cantilever with $70 \mathrm{~dB}$ acoustic noise leading to interesting perspective for high precision micromanipulation tasks in harsh environments.

\section{ACKNOWLEDGMENTS}

The authors would like to thank GUIBERT David for cantilevers fabrication.

\section{REFERENCES}

[1] Zubir M. N. M., Shirinzadeh B., "Development of a Compliant-Based Microgripper for Microassembly", IEEE MESA 2008, Beijing, China.

[2] Chu D. T., Creemer J. F., Sarro P. M., "Lateral nano-Newton forcesensing piezoresistive cantilever for microparticle handling", J. Micromech. Microeng, vol. 16, number. 6, pp. 102 - 106, 2006.

[3] Eisinberg A., Tonet O., Dario P., Macri G., Carrozza M. C, "Microfabricated Instruments for Fetal Cardiac Surgery: Experiments on Haptic Tissue Recognition", International Symposium on Haptic Interfaces for Virtual Environment and Teleoperator Systems, Virginia, USA, 2006.

[4] Boudaoud M., Haddab Y., Le Gorrec Y. , "Modelling of a MEMSbased microgripper: application to dexterous micromanipulation", IEEE/RSJ IROS 2010, Taipei, Taiwan.

[5] Guoyong Z., Chee L. T., Dietmar w. h., Etienne B., "Force-controlled automatic microassembly of tissue engineering scaffolds ", J. Micromech. Microeng, vol. 20, number 3, 2010.

[6] Quan Z., Del C. C., Esteban P. J ., Aurelian A., Koivo H. N, "Environmental influences on microassembly", IEEE/RSJ : IROS 2002, Lausanne,Switzerland.

[7] Weck M., Peschke C., Aurelian A., Koivo H. N, "Equipment technology for flexible and automated micro-assembly", Microsystem Technologies, Volume 10, Number 3, 241-246,

[8] Chao A. W., Tigner M., "Handbook of accelerator physics and engineering". World Scientific Publishing, Singapore, 1999. p 650.

[9] Seryi A., Ruland R., Baklakov B., Chupyra A., Erokhin A., Kondaurov M., Medvedko A., Parkhomchuk V., Singatulin S., Shubin E., Lach J., Plant D., Shiltsev V., "Hydrostatic level system for slow ground motion studies at FERMILAB and SLA", Proceedings of the 2001 Particle Accelerator Conference, Chicago, USA, 2001.

[10] Arai H., Hibino H., Okuma Y., Matsuoka M., Kubo T., Yamazaki F., "Estimation of Ground Motion Characteristics and Damage Distribution in Golcuk, Turkey, Based on Microtremor Measurements", Int Conference on Seismic Zonation, Palm Springs, U.S.A., 2000.

[11] Anis Y.H., Cleghorn W.L., Mills J.K., "Modal analysis of microgrippers used in assembly of MEMS devices", International Conference on MEMS, NANO and Smart Systems, Banff, Alberta, Canada, 2005.

[12] Bolzon B., Brunetti L., Geffroy N., Jrmie A., "Study of vibrations and stabilization at the sub-nanometer scale for CLIC final doublets", NANOBEAM'08, Novosibirsk, Russian Federation 2008.

[13] Shapiro R. A, Berland T., "Noise in the operating room". New England Journal of Medicine, pp. 287:1236-8, 1972.

[14] Ku S., Salcudean S.E., "Design and control of a teleoperated microgripper for microsurgery", IEEE ICRA 1996, Minneapolis, USA.

[15] Jain R. K., Patkar U. S., Majumdar S.,"Micro gripper for micromanipulation using IPMCs (ionic polymer metal composites)", Journal of Scientific and Industrial Research, vol. 68, pp. 23-28, 2009.

[16] Rakotondrabe M., Haddab Y., Lutz P., "Quadrilateral Modelling and Robust Control of a Nonlinear Piezoelectric Cantilever", IEEE trans on control systems technology, vol. 17, no. 3, pp. 528-539, 2009.

[17] Welch P., "The Use of Fast Fourier Transform for the Estimation of Power Spectra: A Method Based on Time Averaging Over Short, Modified Periodograms", IEEE Transactions on Audio Electroacoustics, vol. 15, issue. 2, pp. 70-73, 1967.

[18] Peter G. N., "Understanding and Measuring Noise Sources in Vibration Isolation Systems", VP/CTO Technical Manufacturing Corporation, July 2003.

[19] Ghosh A. K., "Vibration of a cantilever beam on a composite support with base excitation", JSV, vol. 105, number. 1, pp. 91-99, 1986.

[20] Glyn J., "Analysis of beams on elastic foundations using finite difference theory" Thomas Telford Ltd, London, 1997.

[21] Rayleigh L., "Theory of Sound" Dover Publications, New-York, 1877.

[22] Varga, A., "Balancing-Free Square-Root Algorithm for Computing Singular Perturbation Approximations," IEEE CDC 1991, Brighton, UK. 